\title{
Evaluation De La Dégradation De La Chamaeropaie Du Versant Sud Du Mont « jbelLakraa » (Maroc) Etudes Floristique Et Morphométrique
}

\author{
Khalid Benarchid \\ Mohammed Khatori \\ Said Hilali \\ Laboratoire Eco-Conception, Energie, \\ Environnement et Innovation. Faculté des Sciences et Techniques, \\ Université Hassan Premier, Settat - Maroc
}

Doi: 10.19044/esj.2018.v14n14p76 URL:http://dx.doi.org/10.19044/esj.2018.v14n14p76

\begin{abstract}
Our study is devoted to the chamaeropaie degradation assessment of the jbel Lakraa southern slope, located in the northern Middle Atlas in the Ribat Al Khair region. The floristic study revealed a specific richness of 124 species belonging to 95 genera and 33 vascular plants families, as well as a specific diversity relatively high with a Shannon-Weaver index of 3.88. A relatively high specific diversity with a Shannon-Weaver index of 3.88 was observed. However, the floristic composition showed a disturbance expressed by the index of equitability which is 0.58 and a strong therophytisation of $63 \%$ with a very high perturbation index of $69 \%$. This disturbance is also illustrated by the toxic or spiny species proliferation such as Asphodelus ramosus, Drimia maritima and Scolymus hispanicus. The morphometric study was carried out on two strata (stratum 1 anthropized - stratum 2 wooded). The Statistical analysis showed that the diameter and height of the foliage, as well as the number of leaves per individual of the tree stratum are significantly higher than those of the anthropized stratum. Furthermore, an absence of correlations between the morphometric variables was recorded in the anthropized stratum. The chamaeropaie degradation state is alarming as indicated by the floristic and morphometric results, urgent measures must be taken to regulate its exploitation and preservation.
\end{abstract}

Keywords: Biodiversity; Degradation; Floristic; Middle Atlas; Morroco

\section{Résumé}

Notre étude a été consacrée à l'évaluation de la dégradation de la chamaeropaie du versant sud de jbel Lakraa, situé au niveau du Moyen Atlas 
septentrional dans la région de Ribat $\mathrm{Al}$ Khair. L'étude floristique a révélé une richesse spécifique de 124 espèces appartenant à 95 genres et à 33 familles plantes vasculaires, ainsi qu'une diversité spécifique relativement élevée avec un indice de Shannon-Weaver de 3.88. Toutefois la composition floristique a montré une perturbation exprimée par l'indice d'équitabilité qui est de 0,58 et une forte thérophytisation de $63 \%$ avec un indice de perturbation très élevé de $69 \%$. Ce déséquilibre est illustré aussi par la prolifération d'espèces toxiques ou épineuses telles qu'Asphodelus ramosus Drimia maritima et Scolymus hispanicus. L'étude morphométrique a été conduite au niveau de deux strates (strate 1 anthropisée et strate 2 arborée). L'analyse statistique a montré que le diamètre et la hauteur de la frondaison, ainsi que le nombre de feuilles par individu de la strate arborée sont significativement supérieurs à ceux de la strate anthropisée. Par ailleurs, une absence de corrélations entre les variables morphométriques a été enregistrée dans la strate anthropisée. L'état de dégradation de la chamaeropaie est alarmant comme témoignent les résultats floristiques et morphométriques obtenus. Des mesures urgentes doivent doivent être prises pour réglementer son exploitation et sa préservation.

Mots-Clefs: Biodiversité ; Dégradation ; Floristique ; Moyen Atlas ; Maroc

\section{Introduction}

Le rythme de la dégradation des écosystèmes montagneux de la rive sud méditerranéenne a connu une accélération phénoménale au cours de la dernière moitié du vingtième siècle. En plus des changements climatiques, l'impact anthropozoïque demeure la principale cause de cette dégradation, vu que les besoins des populations vulnérables qui vivent au dépend des ressources naturelles dépassent largement la productivité et la résilience des écosystèmes.

Au Maroc, le couvert végétal forestier perd plus de 30000 ha/an suite aux défrichements, aux prélèvements pour les besoins en bois d'énergie et aux incendies (MAMVA, 1995). Des forêts entières ont complètement disparu, laissant place à des formations de dégradation. Le Palmier nain Chamaerops humilis, est une espèce héliophile et thermophile qui trouve son optimum de développement dans les formations de dégradation ou dans les groupements de transition (Barbéro et al. 1981). Il est l'indicateur de l'étage de végétation méditerranéenne semi-aride (Emberger, 1971) mais déborde largement et intensément vers les étages humides tant en montagne que dans les zones littorales. Il présente ainsi une grande plasticité écologique et occupe une large aire géographique au Maroc (Anonyme, 1939).

A l'échelle de notre aire d'étude, le Chamaerops humilis couvre tout le versant sud du mont « Jbel Lakraa » et constitue la nappe de remplacement de la chênaie à Quercus rotundifolia, jadis dense et uniforme. 
D'un point de vue foncier, la partie haute du versant appartient au domaine forestier. Le reste représente des propriétés privées reconnues verbalement avec des limites bien connues et dont la mise en pâturage est libre sans être réglementée. Les droits d'usages sont de coutume et le propriétaire d'un domaine peut mettre en vente le bois présent sur son territoire avec l'accord des services des eaux et forêts. Le paroxysme de cette dégradation a eu lieu pendant les années soixante du siècle dernier, après l'abattage de l'ultime formation de chêne vert pour la fabrication de charbon de bois avec l'arrachage des souches. Les steppes à chamaerops, déjà ancrées, se sont alors étendues sur l'ensemble du versant. D'un point de vue dynamique, la colonisation progressive du Jbel Lakraa par cette essence est la conséquence directe de dégradation de la forêt de chêne vert depuis le début du XX siècle. En effet, ces steppes ne forment jamais un groupement autonome, leur expansion est le résultat de la destruction des forêts dans lesquelles elles étaient un élément constant de remplacement (Anonyme, 1939).

Toutefois, le chamaerops demeure le dernier rempart contre l'érosion, tant il dispose d'un système souterrain puissant, lui permettant de bien fixer le sol et de se régénérer même après avoir été incendié ou coupé au ras du sol. Son port hémisphérique et cespiteux à même le sol, lui a valu son nom de palmier jeté à terre. Il joue des rôles écologiques importants dans la conservation des sols, l'alimentation du bétail et un abri pour la faune. Il a en outre un intérêt socio-économique dans l'artisanat et le pâturage. En effet ses feuilles sont prélevées pour la fabrication de produits artisanaux (feuilles centrales en rames), ou transformées pour la fabrication de crin végétal (feuilles périphériques épanouies). Elles sont aussi régulièrement consommées par le cheptel pendant les périodes durant lesquelles les parcours ne disposent pas suffisamment de plantes fourragères et surtout pendant les années de sècheresse.

Malgré sa rusticité, le Chamaerops humilis est sérieusement menacé. Il est assujetti à une pression anthropozoïque de plus en plus accentuée, surtout après la décision du gouvernement marocain relative à l'interdiction des sachets plastiques et le renouement avec le panier artisanal fait à base de feuilles centrales de Chamaerops.

Parmi les rares études qui ont porté sur les formations à Chamaerops humilis, on peut citer celles effectuées dans la région de Tlemcen en Algérie (Carcia-Castano et al 2014; Medjati, 2014 ; Brahimi et al.2016; Taibi et al.2017).

$\mathrm{Au}$ Maroc, très peu d'études ont porté sur les steppes à Chamaerops. A notre connaissance, mise à part l'étude de Negre (1950) relative à la signification écologique de Chamaerops hmilis aux Beni Moussa, aucune étude n'a été réalisée dans ce sens. En outre Les chamaeropaies suscitent peu 
d'intérêt de la part des pouvoirs publics et ne sont pas encore considérées parmi les priorités de leurs actions de conservation. Il s'avère donc nécessaire d'explorer ces écosystèmes fragiles en dégradation avancée. Le présent travail a donc pour objectifs de contribuer à évaluer l'état des lieux, de prospecter la chamaeropaie, son stade de dégradation ainsi que la biodiversité abritée par cette formation. Ainsi peut-on comprendre son évolution et contribuer à la mise en œuvre d'une stratégie de lutte contre les érosions du sol et de la biodiversité.

Pour répondre à ces objectifs, nous avons réalisé des relevés floristiques afin d' analyser la flore, déterminer la richesse et la diversité spécifique végétale abritée par la chamaeropaie, ainsi que des mesures morphométriques sur des plantes de Chamaerops humilis pour évaluer le degré de leur degradation

\section{Matériel et méthodes}

\section{Présentation de la zone d'étude}

Notre aire d'étude concerne le versant sud du mont «Jbel Lakraa», situé dans le Moyen Atlas septentrional. Elle est délimitée par les coordonnées

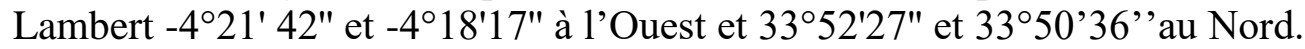
L'altitude y varie de $900 \mathrm{~m}$ à $1621 \mathrm{~m}$ au sommet de jbel Lakraa (Figure 1). L'ensemble est d'une pente raide et d'une exposition sud. L'aire est creusée par de nombreux grands ravinements qui charrient d'importantes quantités de sol, d'éboulis et de gravats surtout au cours des violents orages d'automne. La géologie de ce mont est caractérisée par des formations du lias inférieur et du carixien au niveau de la crête et de celles du toarcien et du bajocien inférieur vers le bas (Sabaoui, 1998). Les « sols », quand ils existent, sont de natures fersiallitiques minces et pauvres en matière organique; ils occupent des surfaces plus ou moins importantes de l'aire d'étude.

Les données climatiques ont été relevées à partir de deux stations météorologiques situées à distances presque égales, à environ $14 \mathrm{~km}$ de notre aire d'étude. Les précipitations ont été relevées de la station de Beni Sohane la plus représentative à notre avis. Elle est située dans la vallée de Zloul qui est délimitée par le versant sud du jbel Lakraa du côté nord. La pluviométrie moyenne annuelle y est de $550 \mathrm{~mm}$, elle est relativement inférieure à celle enregistrée dans la deuxième station Ribat El Khair $(600 \mathrm{~mm})$, située du côté ouest de la vallée Zloul en milieu ouvert au vent de l'Ouest. En effet, La zone d'étude subit un effet de Foehn net et par là elle est plus sèche. Quant aux températures, elles ont été relevées de la station Ribat El Khair puisque la première station n'en dispose pas. La température moyenne du mois le plus chaud atteint $35.46^{\circ} \mathrm{C}$ et la température moyenne du mois le plus froid descend jusqu'à $2.45^{\circ} \mathrm{C}$. L'aire d'étude appartient à l'étage bioclimatique subhumide à hiver frais. Des gelées sévères et des températures négatives peuvent 
subvenir pendant les mois d'hivers et du début de printemps. Les vents froids et humides de l'ouest n'ont pas une influence marquée, quant aux vents du Sud-est (chergui) chauds et secs reviennent assez souvent, surtout pendant le printemps et l'été, ce qui accentue l'aridité et cause souvent un échaudage des cultures. Ils sont particulièrement néfastes à l'agriculture et aux écosystèmes.

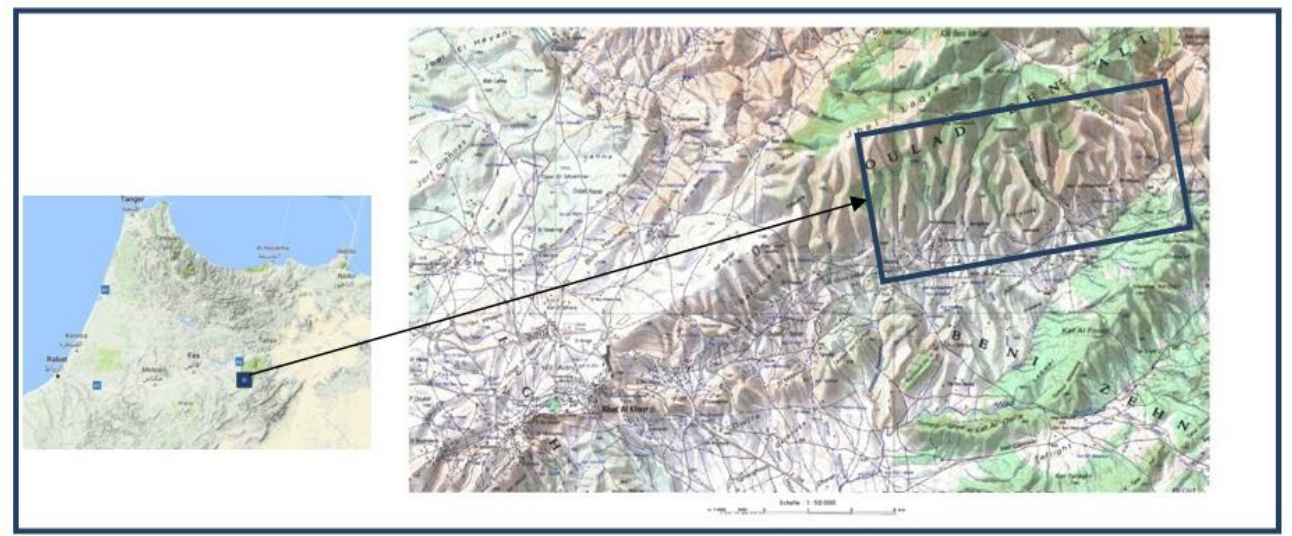

Figure 1 : Localisation de l'aire d'étude : Carte du nord du Maroc et détail de la carte topographique de la région de Ribat $\mathrm{Al}$ Khair.

\section{Etude floristique}

Des relevés floristiques ont été réalisés au niveau d'un transect orienté nord-sud, suivant le flanc du synclinal «Vallée de Zloul » avec un gradient altitudinal décroissant et une forte exposition au soleil le long d'un dénivellement de $700 \mathrm{~m}$. Cette méthode a été utilisée pour recouvrir la diversité maximale des situations variées : topographiques, géologiques, géomorphologiques et végétales. 16 relevés ont été effectués sur des placettes de $100 \mathrm{~m}^{2}$ pendant les mois de mai 2015 et 2016, période durant laquelle la majorité des plantes des strates herbacées et arbustives sont en plein développement. Les plantes ont été déterminées à l'aide de la flore pratique du Maroc (Fennane et al., 1999; Fennane et al., 2007 ; Fennane et al., 2014 ; Dobignard et Chatelain 2010-2013). Chaque espèce est accompagnée par l'indice abondance-dominance de Braun Blanquet (1951). La systématique des taxons adoptée est la classification APG IV 14 (2016).

\section{Diversité floristique}

La diversité floristique a été estimée par l'indice de Shannon-Weaver (H'), qui tient compte de l'abondance de chaque espèce :

$\mathrm{Pi}=\mathrm{ni} / \mathrm{N}$

$$
H^{\prime}=\sum_{i=1}^{s} p i \log _{2} p i
$$

ni : Recouvrement de l'espèce i dans le relevé ; 
$\mathrm{N}$ : Somme des recouvrements de l'ensemble des espèces $\mathrm{S}$.

L'indice Shannon-Weaver est souvent complété par l'indice d'équitabilité de Pielou (E). Ce dernier renseigne sur la structure d'abondance relative des espèces ou sur leur distribution, il est exprimé par la formule suivante:

H': Indice Shannon-Weaver;

$$
E=\frac{\mathbf{H}^{\prime}}{\log _{2} S}
$$

$\mathbf{S}:$ Nombre total des espèces.

\section{Indice de perturbation}

L'indice de perturbation (IP) établi par (Loisel et Gomila 1993), permet de quantifier le degré de perturbation de la végétation. Il est calculé par la formule ci-dessous :

$$
I P=\frac{\text { Chaméphytes }+ \text { Thérophytes }}{\text { Nombre total des espèces }}
$$

\section{Etude morphométrique}

Afin d'évaluer le degré de dégradation des plantes de Chamaerops humilis, des mesures morphométriques ont été effectuées au cours du mois de septembre 2016. Elles ont porté sur :

* Le nombre de feuilles ;

* La hauteur de la frondaison ;

* Le diamètre de la frondaison ;

* Le nombre de rejets par souche.

Un échantillonnage stratifié aléatoire non proportionnel a été adopté pour pouvoir évaluer l'impact anthropozoïque. Ainsi 2 strates ont été distinguées :

$\checkmark \quad$ Strate 1 (anthropisée): Elle représente la grande partie de l'aire d'étude où la forêt de chêne vert a totalement disparu.

$\checkmark \quad$ Strate 2 (arborée) : Elle représente quelques îlots relictuels de chêne vert témoins de l'existence antérieure de la forêt de chêne vert. Ils se situent généralement au niveau de flancs de grands talwegs relativement accidentés et moins anthropisés.

Les observations ont été effectuées sur des individus choisis au hasard. Le nombre d'individus par strate a été choisi en tenant compte de leurs tailles respectives et aussi de manière à pouvoir observer correctement la strate 2 dont l'aire est largement inférieure à celle de la strate 1. Ainsi, 100 individus ont été observés pour la strate 1 et 50 individus pour la strate 2 .

Les 2 échantillons vérifient les conditions de normalité et d'homogénéité des variances pour les 4 variables. Les mesures observées ont 
été traitées statistiquement par le test de Student (t) pour comparer leurs moyennes au niveau des 2 strates.

Les corrélations entre les différentes variables ont été déterminées par la méthode de la régression linéaire. Elles permettent de définir les relations qui existent entre elles et de pouvoir les comparer au sein des deux strates. Les traitements statistiques ont été réalisés par le logiciel IBM SPSS Statistics 22.

\section{Résultats}

\section{Etude floristique}

La richesse spécifique de notre aire est de 124 espèces appartenant à 95 genres et à 33 familles de plantes vasculaires. La figure 2 illustre la dominance de la famille des Asteraceae qui compte à elle seule $27 \%$ de la richesse spécifique, suivie de celle des Poaceae avec $13 \%$, de celle des Fabaceae et des Lamiaceae avec pour chacune 8\%, puis de celle des Apiaceae, Caryophyllaceae et Brassicaceae avec $5 \%$ chacune. 19 familles sont monospécifiques.

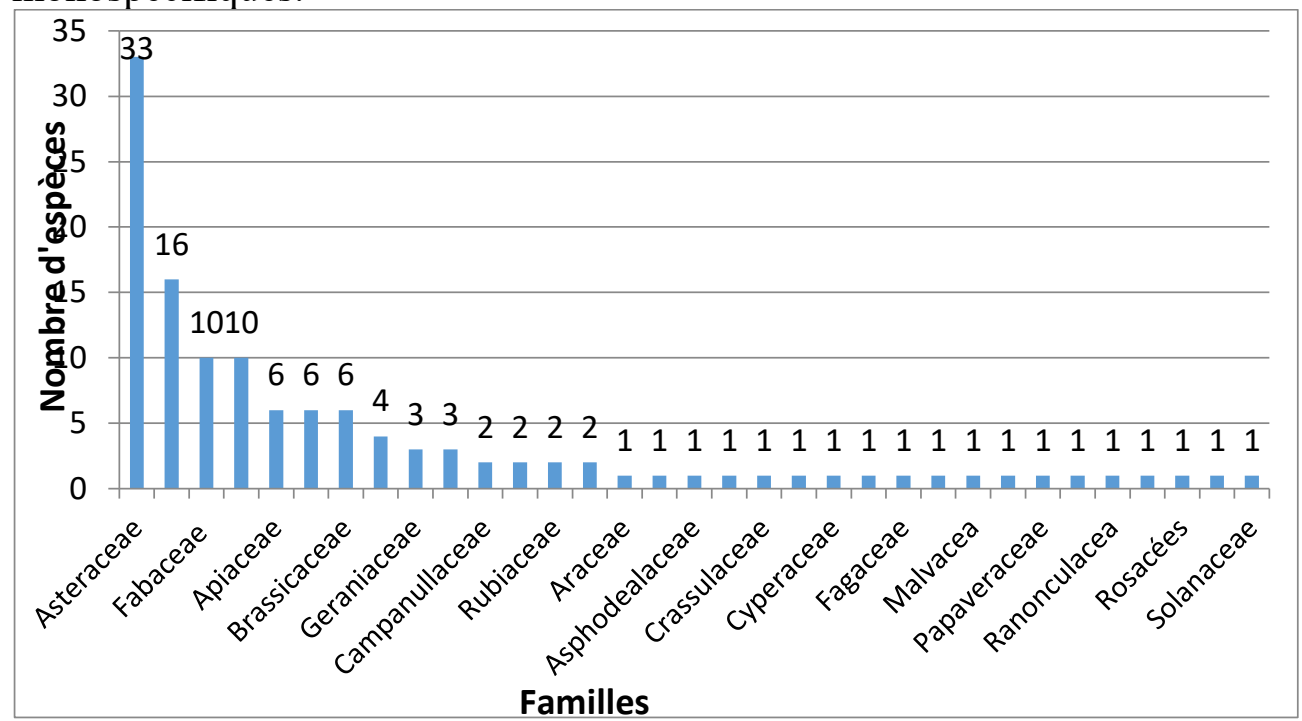

Figure 2: Distribution des espèces par familles de plantes vasculaires.

\section{Indices de diversité}

La diversité spécifique aété évaluée par la mesure des indices de Shannon-Weaver (H') et d'équitabilité (E) qui sont respectivement de 3.72 et 0.54 .

\section{Spectre biologique}

Les plantes relevées au niveau de la chamaeropaie de Jbel Lakraa ont été classées selon leurs types biologiques (Raunkiaer, 1934). Les thérophytes dominent largement avec un pourcentage de $63 \%$, suivis des 
hémicryptophytes avec $23 \%$, alors que les chaméphytes, les géophytes et les phanérophytes ne totalisent que $14 \%$ (figure 3 ).

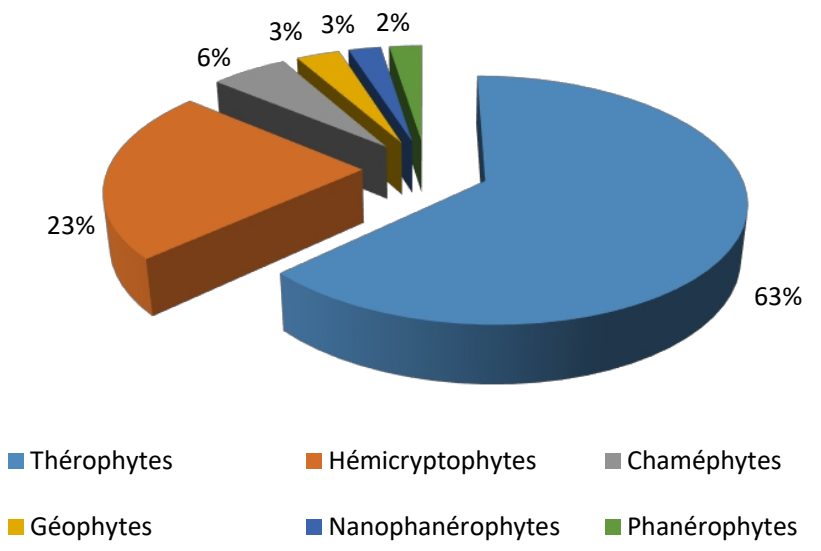

Figure 3 : Spectre biologique des espèces inventoriées dans la chamaeropaie.

\section{Indice de perturbation IP}

L'indice de perturbation IP calcule le pourcentage des chaméphytes et des thérophytes par rapport au nombre total des espèces. Il est de $69 \%$ pour notre aire d'étude.

\section{Etude morphométrique}

\section{Comparaison des moyennes}

Les mesures des variables morphométriques ont été effectuées sur 100 individus pour la strate 1 anthropisée et 50 individus pour la strate 2 arborée. Les résultats montrent une nette différence entre les deux strates pour le diamètre et la hauteur de la frondaison ainsi que pour le nombre de feuilles (tableau1). Le traitement statistique par le test $t$ de Student a montré que les moyennes de ces trois variables de la station 2 sont significativement supérieures à celles de la station 1. Alors qu'il n'existe pas de différence significative entre les deux strates concernant le nombre de rejets par souche.

Tableau 1 : Mesures moyennes des variables morphométriques du Chamerops humilis.

\begin{tabular}{|c|c|c|c|c|}
\hline Strates & $\begin{array}{c}\text { Diamètre } \\
\text { Frondaison }(\mathrm{cm})\end{array}$ & $\begin{array}{c}\text { Hauteur } \\
\text { Frondaison } \\
(\mathrm{cm})\end{array}$ & $\begin{array}{c}\text { Nombre } \\
\text { feuilles par } \\
\text { individu }\end{array}$ & $\begin{array}{c}\text { Nombre } \\
\text { rejets par } \\
\text { souche }\end{array}$ \\
\hline Strate 1 & 46,01 & 44,53 & 9,62 & 5,56 \\
\hline Strate2 & 75,42 & 63,52 & 12,88 & 5,8 \\
\hline $\begin{array}{c}\text { Moyenne } \\
\text { générale }\end{array}$ & 60,715 & 54,025 & 11,25 & 5,68 \\
\hline
\end{tabular}




\section{Régression linéaire}

Le tableau 2 des corrélations montre que pour les deux strates, le nombre de rejets par souche varie indépendamment des autres variables. Les coefficients de corrélation sont quasi nuls. Alors que le diamètre et la hauteur de la frondaison sont fortement corrélés, avec un coefficient R de 0.91 .

Cependant, les corrélations entre les couples de variables (Diamètre / Nombre de feuilles) et (Hauteur / Nombre de feuilles) diffèrent entre les deux strates. La corrélation est forte entre ces deux couples au niveau de la strate 2 avec des coefficients respectifs de 0.72 et 0.63 . Elle est faible pour la strate 1 avec des coefficients respectifs de 0.41 et 0.42 . Tableau 2 : Tableau de corrélations des variables morphométriques du Chamerops humilis.

\begin{tabular}{|c|c|c|}
\hline \multirow{2}{*}{ Désignations } & \multicolumn{2}{|c|}{ Coefficient de corrélation R } \\
\cline { 2 - 3 } & Strate 1 & Strate 2 \\
\hline Diamètre / Hauteur & 0.91 & 0.91 \\
\hline Diamètre / Nombre feuilles & 0.41 & 0.72 \\
\hline Diamètre / Nombre rejets & 0.26 & 0.24 \\
\hline Hauteur /Nombre feuilles & 0.42 & 0.63 \\
\hline Hauteur / Nombre rejets & 0.24 & 0.17 \\
\hline Nombre feuilles / Nombre rejets & 0.07 & 0.11 \\
\hline
\end{tabular}

\section{Discussion}

\section{Etude floristique}

\section{Indice de Shannon-Weaver $\left(\mathrm{H}^{\prime}\right)$}

L'étude floristique a montré une richesse floristique de 124 espèces. Ce sont les mêmes familles qui dominent et respectivement avec le même ordre d'importance dans les compositions des chamaeropaies des monts de Tlemcen en Algérie (Taibi et al., 2017). La diversité spécifique a été évaluée par la mesure de l'indice de Shannon-Weaver ( $\left.\mathrm{H}^{\prime}\right)$ : Quand cet indice est élevé cela correspond à des conditions environnementales favorables à l'installation d'un grand nombre d'espèces représentées par un petit nombre d'individus. Il est admis que dans la réalité, l'indice de Shannon prend souvent des valeurs comprises entre 1 et 5 .

Contrairement à ce qu'on s'attendait, la valeur de notre indice est de 3.72. Elle peut être considérée comme relativement élevée, et reflète ainsi l'importance de la diversité spécifique abritée par la Chamaeropaie. En effet, beaucoup d'espèces ont été relevées sous des touffes vigoureuses de Chamaerops humilis. Elles sont protégées par leurs feuilles et leurs pétioles épineux contre le bétail. Elles profitent aussi d'une sorte de microclimat relativement humide qui les protège contre l'intensité du rayonnement solaire durant les saisons chaudes. 


\section{Indice d'équitabilité (E)}

L'indice d'équitabilité (E) complète l'indice de Shannon-weaver. Il renseigne sur la structure d'abondance relative des espèces ou leurs distributions. Cet indice varie entre 0 et 1 . Une distribution équitable est exprimée par un indice proche de 1 et inversement, une distribution dominée par une seule espèce tend vers 0 . La valeur de l'indice d'équitabilité de notre aire d'étude est de 0.54 . Il reflète une distribution perturbée des espèces. Cette anomalie de la composition floristique pourrait être expliquée par la dominance d'espèces expansionnistes essentiellement Chamaerops humilis, Asphodelus microcarpus et des graminées comme Aegilops neglecta et Hordeum murinum. La prolifération des Poaceae qui représente $13 \%$ de la richesse spécifique pourrait être considérée comme un indicateur de surpâturage (Ghiloufi et al., 2015; Catorci et al., 2011). En effet, la mise en défens des parcours dans la forêt de Maamora au Maroc, à titre d'exemple, a permis une diminution de la biomasse des Poaceae et une augmentation de celle des Astéraceae, des Fabaceae et d'autres familles (Abourouh et al,. 2005).

\section{Spectre biologique}

Le spectre biologique de notre aire d'étude montre une forte thérophytisation soit $63 \%$. Celle-ci est plus accentuée que celle enregistrée dans les chamaropaies de la région de Tlemcen en Algérie dont le pourcentage moyen en thérophytes était de 59\% (Taibi et al., 2017 ).

La thérophytie peut être considérée comme une forme d'adaptation naturelle des plantes aux contraintes climatiques telles que le froid hivernal ou la sécheresse estivale (Daget, 1980 ; Barbero et al., 1990). Elle est aussi une réponse à un pâturage excessif. Dans le contexte de notre étude, elle reflète surtout le degré de dégradation de la chamaeropaie. En effet, elle est considérée par Quézel (2000) comme un stade ultime de dégradation de la végétation.

\section{Indice de perturbation IP}

L'indice de perturbation IP de notre aire d'étude est de 69\%. Il est aussi élevé que ceux obtenus par El Hamrouni (1992) 70\% et Benkhettou (2015) $63,3 \%$. Il reflète un degré important de déséquilibre et de perturbation du milieu qui pourrait être exprimé aussi par la forte thérophytie (63\%) enregistrée par le spectre biologique. En effet, selon Grime (1977), la stratégie rudérale d'espèces thérophytes est une réponse adoptée par les plantes dans un milieu perturbé.

La perturbation du milieu pourrait être expliquée essentiellement par le surpâturage. Ainsi ce site est toujours connu par un pâturage intense et une pression humaine sur le couvert végétal. La chamaeropaie est ouverte toute 
l'année au pâturage. Aucune réglementation n'est adoptée pour permettre aux plantes d'achever leur cycle de développement et de reconstituer au sol le stock de semences nécessaire à leur régénération. La pratique de la mise en défens temporaire appelée 'Agdal', jadis commune partout au Maroc, permet le maintien d'une plus grande diversité floristique et assure la durabilité des parcours (Genin et al., 2012). La perturbation du site pourrait être illustrée aussi par la prolifération de l'espèce expansionniste Chamaerops humilis, d'espèces toxiques pour le bétail, Asphodelus ramosus, Drimia maritima et de l'espèce épineuse Scolymus hispanicus. Elles constituent toutes des espèces d'un processus lent de remplacement d'une forêt en dégradation.

\section{Etude morphométrique Comparaison des moyennes}

Les faibles valeurs observées dans la strate 1 pourraient traduire l'intensité de la pression anthropozoïque sur cette aire de la chamaeropaie. En effet les feuilles périphériques étalées sont régulièrement fauchées et transformées dans une unité de fabrication de crin végétal installée dans le secteur. Celle-ci est la seule unité qui a survécu parmi environ 50 unités implantées au Maroc depuis les années 20 du siècle dernier. Par ailleurs les feuilles sont cisaillées par le bétail au niveau des parties apicales et très recherchées par la population avoisinante pour la fabrication de produits artisanaux. Ces pratiques donnent des frondaisons rabougries et moins développées et pourraient causer à la longue un épuisement des souches qui deviennent plus vulnérables aux stress environnementaux et par la suite leur éventuelle dégénérescence.

L'état alarmant de dégradation de la chamaeropaie dans la strate 1, pourrait être illustré par des surfaces complètement dénudées par l'érosion hydrique suite à la dégénérescence des souches de Chamaerops (Photo1). Par contre, le contraste est frappant pour les souches de la states 2 qui sont vigoureuses avec un taux de couverture supérieur à $50 \%$ (photo 2 ).

\section{Régression linéaire}

Les faibles corrélations obtenues entre les couples de variables (Diamètre de la frondaison / Nombre de feuilles) et (Hauteur de la frondaison / Nombre de feuilles) dans la strate 1 sont respectivement de 0.41 et 0.42 , elles pourraient contraster aussi bien avec celles obtenues dans la strate 2 qui sont respectivement de 0.72 et 0.63 et celles obtenues par les travaux de Hasnaoui (1998) qui sont respectivement de 1 et 0,83 . Normalement, le diamètre devrait croître avec le nombre de feuilles. En effet, fort probablement cela est dû essentiellement à la pression anthropozoïque que connait la strate1.

Il convient de souligner que la pression sur la chamaeropaie a repris fortement après la décision du gouvernement marocain relative à l'interdiction 
des sachets plastiques et leur substitution par des produits alternatifs entrée en vigueur à partir du 1er juillet 2016. Effectivement, après deux mois de cette décision, le prix moyen de vente d'une unité de Mezlag (= 3 rangées superposées, d'un mètre de long, de feuilles centrales non encore déployées) a augmenté d'au moins 30\%, il a passé de 30 à 45 Dirhams.

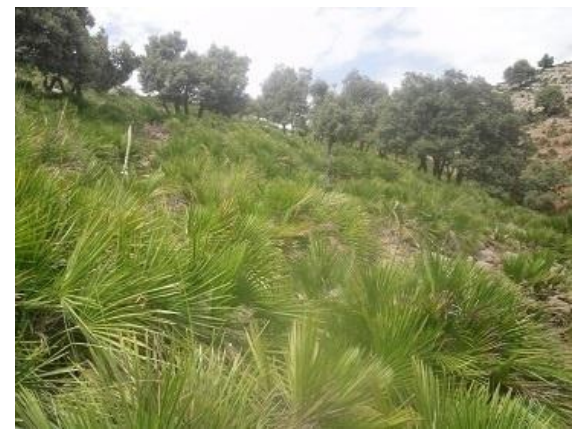

Photo 1 : Ilots relictuels de chêne vert abritant des après touffes de Chamaerops humilis bien développées (strate 2).

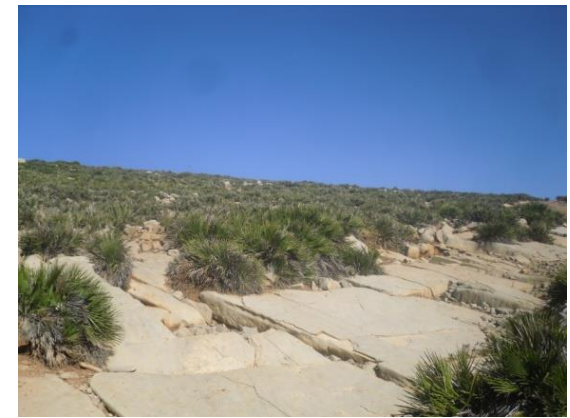

Photo 2 : Surfaces complètement dénudées la disparition des touffes de Chamaerops humilis (strate 1)

\section{Conclusion}

Le présent travail a eu pour objectifs l'évaluation de la diversité floristique de la chamaeropaie du versant sud du mont jbel Lakraa, ainsi que l'état de sa dégradation. Les résultats de l'étude floristique révèlent une richesse spécifique de 124 espèces appartenant à 95 genres et à 33 familles de plantes vasculaires, ainsi qu'une diversité spécifique relativement élevée avec un indice de Shannon de 3.72. Toutefois, la dominance d'espèces expansionnistes telles que Chamaerops humilis, Asphodelus ramosus et d'autres graminées montrent une perturbation du milieu. Cette perturbation a été confirmée par l'indice d'équitabilité qui est de 0,54. Par ailleurs, l'analyse du spectre biologique montre une dominance des thérophytes avec $63 \%$, qui marque une forte thérophytisation exprimée par un indice de perturbation élevé de $69 \%$.

La dégradation de la chamaeropaie pourrait être illustrée aussi par la prolifération d'espèces toxiques ou épineuses telles que, Asphodelus ramosus, Drimia maritima et Scolymus hispanicus.

L'étude morphométrique confirme l'impact anthropozoïque sur la dégradation de la chamaeropaie. La comparaison des moyennes par le test de Student montre que le diamètre et la hauteur de la frondaison, ainsi que le nombre de feuilles par individu de la strate 2 (arborée) sont significativement supérieurs à ceux de la strate 1 (anthropisée). En outre, à l'exception du couple (diamètre / hauteur), il n'existe pas de corrélation entre les variables au niveau de la strate anthropisée. 
L'état de dégradation de la chamaeropaie du versant sud de jbel Lakraa est alarmant comme en témoignent à la fois l'érosion avancée de la biodiversité et autant sur l'épuisement des sols. Néanmoins, notre étude a porté sur une aire géographique restreinte. L'exploration d'autres chamaeropaies dans des conditions environnementales différentes est donc nécessaire pour mieux appréhender leur diversité et leur état de dégradation. Par ailleurs, l'étude histométrique pourrait nous éclairer aussi sur l'adaptation de cette espèce aux conditions environnementales locales.

\section{Remerciements}

Nous remercions sincèrement les Professeurs Mohamed IBN TATTOU et Mohamed FENNANE de l'Institut Scientifique de Rabat, ainsi que Professeur Cyrilles CHATELAIN du Conservatoire et jardin botaniques de Genève de bien vouloir contribuer à l'identification des espèces relevées dans le cadre de ce travail. Nous tenons aussi à remercier le Professeur de la langue française Mostapha TENNICH et le Professeur de la langue anglaise Abdelali AGHBALOU qui ont bien voulu relire et coorriger le présent document.

\section{References:}

1. Abourouh, M., Taleb, M., Makhloufi, M., Boulmane, M., et Aronson J.(2005). Biodiversité et dynamique de la végétation dans la subéraie de la Maâmora : Effet de la durée de clôture. Forêt méditerranéenne. Vol 26. $n^{\circ} 4: 275-285$.

2. Anonyme.(1939). La distribution géographique de quelques espèces tenant une place importante dans la végétation du Maroc. Publications de l'Institut géobotanique Rübel à Zurich: 150-153.https://www.eperiodica.ch/cntmng?pid=gbi-001:1939:14::191( consulté le 11/03/2018).

3. Barbero, M., Loisel, R., et Quezel, P.(1990). Les apports de la phytoécologie dans l'interprétation des changements et perturbation induite par l'Homme sur les écosystèmes forestiers méditerranéens. Forêt Méditerranéenne XII, 3: 194-216.

4. Barbéro, M., Quézel, P., et Rivas-Martínez, M.(1981). Contribution à l'étude des groupements forestiers et préforestiers du Maroc. Phytocoenologia, vol. 9, $\mathrm{n}^{\circ} 3: 311-412$.

5. Benkhettou, A., Azouzi, B., Djili K, Benkhettou, M., Zedek, M., et Saadi, R.(2015). Diversité floristique du massif du Nador en zone steppique (tiaret, Algérie). European Scientific Journal vol. 11, No21:401- 419.

6. Brahimi, N., Hasnaoui, O., et Cherif, I. (2016). Contribution of Chamaerops humilis L. (Arecaceae) in conservation in situ of soil 
resources in Tlemcen region (Western Algeria). Open Journal of Ecology, 6: 562-567.

7. Braun-Blanquet, J. (1951).Planzen soziologie, Springer Ed. Vienne. $2^{\circ}$ Ed. 631p.

8. Carcia-Castano, J.L., Terrab, A., Ortiz, A., Stuessy et Talavera, S. (2014). Patterns of phylogeography and vicariance of Chamaerops humilis L. (Palmae). Turkish J. Bot. 38: 1132-1146.

9. Catorci, A., Ottaviani, G., Ballelli, S., et Cesaretti, S.(2011). Functional differentiation of central Apennine grass lands undermowing and grazing disturbance regimes. Pol.J.Ecol.59: 115128.

10. Daget, P. 1980. Sur les types biologiques en tant que stratégie adaptative (cas des thérophytes), Recherches d'écologie théorique, les stratégies adaptatives, Maloines, Paris : 89-114.

11. Dobignard, A., et Chatelain, C. (2010-2013). Index synonymique. Flore Afrique du Nord, Vol.1-5. Edit. Conservatoire et Jardin botaniques de la ville de Genève.

12. El-Hamrouni, A.(1992). Végétation forestière et préforestière de la Tunisie: typologie et éléments pour la gestion. Thèse Doctorat. Université Aix-Marseille III. 220 p.

13. Emberger,L.(1971). Considérations complémentaires au sujet des recherches bioclimatiques et phytogéographiques-écologiques. In: Travaux de botanique et d'écologie. Masson et Cie et (éd.) Paris. 291301.

14. Fennane, M., Ibn Tatou, M., Mathez, J., Ouyahia, A., et El Oualidi, J. 1999. Flore pratique du Maroc.Vol. I. Institut Scientifique. Université Mohamed V. Agdal. Rabat.

15. Fennane, M., Ibn Tatou, M., Ouyahia, A., et El Oualidi, J. 2007. Flore pratique du Maroc. Vol I1. Institut Scientifique. Université Mohamed V. Agdal. Rabat.

16. Fennane, M., Ibn Tatou, M., et El Oualidi, J. 2014. Flore des effets de débroussaillement pratique du Maroc. Vol I1 I. Institut Scientifique. Université Mohamed V. Agdal. Rabat.

17. Genin, D., Kerautret, L., Hammi, S., Cordier, J.B., et Al ifriqui, M.(2012). Biodiversité et pratiques d'Agdal. Un élément de l'environnement à l'épreuve de ses fonctions d'utilité pour les sociétés du Haut-Atlas cen-tral. In Auclair, L., Al ifriqui, M.(dir.) : Agdal . Patrimoine socio-éco-logique de l'Atlas marocain, Rabat, IRDIRCAM. Rabat Maroc: 93-127.

18. Ghiloufi, W., Quéro Pérez, J.L., García-Gómez, M., Chaieb, M.(2015). Assessment of species diversity and state of Stipa tenacissima steppes.Turk.J.Bot.39: 227-237. 
19. Grime, J.P. (1977). Evidence for the existence of three primary strategies in plants and its relevance to ecological and Evolutionary theory. The American Naturalist 111 (982) : 1169-1194.

20. Hasnaoui, O.(1998). Etude des groupements à Chamaerops humilis L. argenteadans la région de Tlemcen. Thèse Magister. Université Abou Bakr Belkaid. Tlemcen.

21. Loisel, R., et Gomila, H. (1993). Traduction sur les écosystèmes forestiers et préforestiers par un indice de perturbation. Ann.Soc.Sci. Nat. Archeol. 42(2) : 123-132.

22. MAMVA. (1995). Direction des Eaux et Forêts et de la Conservation des Sols, La désertification au Maroc : Causes, ampleur et réalisations. Terre et Vie : $15-16 ; 17$.

23. Medjati, N. (2014). Contribution à l'étude biologique et phytoécologique du Chamaerops hmilis L., dans la partie occidentale de l'Algérie. Thèse Doctorat Université Abou Bakr Belkaid. Tlemcen.

24. Nègre, R. (1950). Signification écologique du Doum (Chamaerops humilis L.) aux Beni Moussa. C.R. Séances Mens. Soc. Sci. Nat. Maroc, Rabat. 2: 25-27.

25. Quézel, P.(1983). Flore et végétation de l'Afrique du Nord, leur signification en fonction de l'origine, de l'évolution et des migrations des flores et structures de végétations passées. Bothalia(14): 411-416.

26. Quézel, P.(2000). Réflexions sur l'évolution de la flore et de la végétation au Maghreb méditerranéen. Ibis Press Edit., Paris.

27. Raunkiaer, C.(1934). The life forms of plants and statistical plant. Geography. Claredonpress. Oxford. 632 p.

28. Sabaoui, A.(1998). Rôle des inversions dans l'évolution mésocénozoique du Moyen Atlas septentrional (Maroc). L'exemple de la transversale El Menzel-Ribat Al Khair-Bou Iblane. Thèse Doctorat. Université Mohamed V. Rabat.

29. Taibi, A., Hasnaoui, O., et Medjati, N. 2017. Caractérisation biologique et biogéographique des Chamaeropaies dans les monts de Tlemcen (Algérie occidentale). International Journal of Innovation and Applied Studies Vol. 19 No. 3:648-653. 